Correspondence John P. Morrissey j.morrissey@ucc.ie

\title{
Pseudomonas aeruginosa secreted factors impair biofilm development in Candida albicans
}

\author{
Lucy J. Holcombe, ${ }^{1} \dagger$ Gordon McAlester, ${ }^{1} \dagger$ Carol A. Munro, ${ }^{3}$ \\ Brice Enjalbert, ${ }^{3}$ Alistair J. P. Brown, ${ }^{3}$ Neil A. R. Gow, ${ }^{3}$ Chen Ding, ${ }^{4}$ \\ Geraldine Butler, ${ }^{4}$ Fergal O'Gara ${ }^{1,2}$ and John P. Morrissey ${ }^{1}$ \\ ${ }^{1}$ Department of Microbiology, University College Cork, Cork, Ireland \\ ${ }^{2}$ BIOMERIT Research Centre, University College Cork, Cork, Ireland \\ ${ }^{3}$ Institute of Medical Sciences, University of Aberdeen, Aberdeen, UK \\ ${ }^{4}$ School of Biomolecular and Biomedical Science, Conway Institute, University College Dublin, \\ Dublin 4, Ireland
}

\begin{abstract}
Signal-mediated interactions between the human opportunistic pathogens Pseudomonas aeruginosa and Candida albicans affect virulence traits in both organisms. Phenotypic studies revealed that bacterial supernatant from four $P$. aeruginosa strains strongly reduced the ability of C. albicans to form biofilms on silicone. This was largely a consequence of inhibition of biofilm maturation, a phenomenon also observed with supernatant prepared from non-clinical bacterial species. The effects of supernatant on biofilm formation were not mediated via interference with the yeast-hyphal morphological switch and occurred regardless of the level of homoserine lactone (HSL) produced, indicating that the effect is $\mathrm{HSL}$-independent. A transcriptome analysis to dissect the effects of the $P$. aeruginosa supernatants on gene expression in the early stages of C. albicans biofilm formation identified 238 genes that exhibited reproducible changes in expression in response to all four supernatants. In particular, there was a strong increase in the expression of genes related to drug or toxin efflux and a decrease in expression of genes associated with adhesion and biofilm formation. Furthermore, expression of YWP1, which encodes a protein known to inhibit biofilm formation, was significantly increased. Biofilm formation is a key aspect of $C$. albicans infections, therefore the capacity of $P$. aeruginosa to antagonize this has clear biomedical implications.
\end{abstract}

\section{INTRODUCTION}

Although the bacterium Pseudomonas aeruginosa and the yeast Candida albicans are benign members of the mucosal flora of some healthy individuals, they are also serious opportunistic pathogens of susceptible individuals. $P$. aeruginosa, for example, is the leading cause of mortality in cystic fibrosis (CF) patients and C. albicans is the most common hospital-acquired fungal infection. In some cases,

†These authors contributed equally to this work.

Abbreviations: 30C12HSL, 3-oxo-C12-homoserine lactone; CF, cystic fibrosis; CGD, Candida Genome Database; CLSM, confocal laser scanning microscopy; FCS, fetal calf serum; HSL, homoserine lactone; LB, Luria-Bertani; LSD, least significant difference; SAM, significance analysis of microarrays.

Complete expression data for the analysis of changes in gene expression under different test conditions have been submitted to the EBI database under accession number E-MEXP-1614.
Candida and Pseudomonas have been co-isolated from the CF lung or serious burn wounds (Bakare et al., 2003; Gupta et al., 2005; Valenza et al., 2008) but the degree to which they communicate in vivo remains largely unknown. A number of recent studies, however, found that these organisms can interact (El-Azizi et al., 2004; Kerr, 1994; Nseir et al., 2007) and that these complex interactions are mediated, at least in part, by small signal molecules (Cugini et al., 2007; De Sordi \& Mühlschlegel, 2009; Hogan \& Kolter, 2002; Hogan et al., 2004; McAlester et al., 2008; Storey et al., 1998; Wargo \& Hogan, 2006). The Pseudomonas quorum sensing signal 3-oxo-C12-homoserine lactone (3OC12HSL), which is a regulator of bacterial virulence factor production (Shiner et al., 2005), inhibits hyphal development in C. albicans when present in culture supernatants or added exogenously (McAlester et al., 2008). This morphological effect is likely to reduce the virulence of $C$. albicans as the ability to switch between yeast and hyphae is an important virulence trait (Gow et al., 2002; Whiteway \& Oberholzer, 2004). Conversely, 
farnesol, which is a quorum sensing (QS) molecule produced by $C$. albicans to control hyphal development (Hornby et al., 2001), reduces Pseudomonas quinolone signal production and swarming motility, two traits important for virulence in $P$. aeruginosa (McAlester et al., 2008).

The effects of Peudomonas signals on Candida biofilm formation are of particular interest as many C. albicans infections are the result of drug-resistant biofilm formation on surfaces of indwelling medical devices, such as catheters and heart valves (d'Enfert, 2006; Kojic \& Darouiche, 2004; Nobile \& Mitchell, 2006). Mature biofilm formation in $C$. albicans requires the ability to transform from yeast to hyphal growth (Baillie \& Douglas, 1999; Ramage et al., 2002a, b); therefore, microbial interactions that interfere with this process may also modulate biofilm formation and aid in the identification of molecular targets for therapeutic intervention.

Our previous work established that supernatants from some clinical isolates of $P$. aeruginosa inhibit morphological development in C. albicans in a 3OC12HSLdependent fashion (McAlester et al., 2008). In this study, we used targeted phenotypic assays to specifically assess the effects of bacterial supernatants on Candida adhesion and biofilm formation. Following this, global gene expression was examined to establish the range of effects that $P$. aeruginosa supernatants/signals have on C. albicans developmental processes.

\section{METHODS}

Strains and culture conditions. All strains used in this study are listed in Supplementary Table S1, available with the online version of this paper. The P. aeruginosa CF isolates (CF144 and CF177) have been genetically characterized previously by our group and shown to be genotypically distinct (Adams et al., 1998; Finnan et al., 2004). C. albicans DAY 185, BWP 17 and BCa2-10 were kind gifts from A. Mitchell, B. Granger and A. Johnson, respectively. All bacterial strains were routinely cultured at $37^{\circ} \mathrm{C}$ in Luria-Bertani (LB) broth or on LB plates. Antibiotics for selection of PAO1 $\Delta \mathrm{QS}$ were used at the following concentrations: $100 \mu \mathrm{g}$ gentamicin $\mathrm{ml}^{-1}$ and $200 \mu \mathrm{g}$ tetracycline $\mathrm{ml}^{-1}$. C. albicans was grown in nonfilament-inducing medium YNB $[1 \times$ YNB salts with ammonium sulphate (Difco 291940), $0.2 \%$ glucose and $0.1 \%$ maltose] and filament-inducing medium YNBNP [YNB supplemented with $25 \mathrm{mM}$ phosphate buffer ( $\mathrm{pH} 7$ ) and $2.5 \mathrm{mM} \mathrm{N}$-acetylglucosamine (Sigma A-8625)] as described previously (McAlester et al., 2008).

Bacterial supernatant preparation. All bacterial supernatants were prepared essentially as described previously (McAlester et al., 2008). Briefly, after $16 \mathrm{~h}$ growth in LB medium, cells were harvested by centrifugation and the resulting supernatant was sterilized by filtration. The sterile supernatant was immediately lyophilized and resuspended at a concentration of $20 \times$ in sterile pure water. Supernatants were used immediately or stored for short periods of time at $-80{ }^{\circ} \mathrm{C}$. As a control, sterile LB media was filtered, lyophilized and resuspended to a concentration of $20 \times$ in the same manner as the bacterial samples.
Treatment of C. albicans with bacterial supernatants. C. albicans cells were grown as yeast cultures overnight at $30{ }^{\circ} \mathrm{C}$ in YNB medium. Yeast cells were diluted in YNBNP filament-inducing medium to $\mathrm{OD}_{600} 0.05$ and incubated at $37^{\circ} \mathrm{C}$. Upon inoculation from YNB to filament-inducing medium, sterile concentrated bacterial supernatant was added at a final concentration of $2 \times$ from a $20 \times$ stock. Incubation was then continued for biofilm or transcriptome analysis experiments, as described below.

Yeast biofilm analysis. Biofilms were grown on silicone squares essentially as described previously (Ding \& Butler, 2007; Nobile et al., 2006). Briefly, silicone squares $(1.5 \times 1.5 \mathrm{~cm})$ were cut from sheets (Cardiovascular Instruments) and autoclaved. The squares were then pre-treated with fetal calf serum (FCS) in a 12-well plate and incubated with shaking at $37^{\circ} \mathrm{C}$ overnight. The silicone was washed with PBS buffer to remove the residual FCS and moved to a new 12well plate. C. albicans cells were treated with bacterial supernatant in YNBNP as described above and $3 \mathrm{ml}$ cultures were added to the 12well plate containing the pre-treated silicone. The plate was incubated on a rotor at $37^{\circ} \mathrm{C}$ for $2 \mathrm{~h}$ (initial adhesion phase) with gentle shaking. The medium was removed, and the silicone was gently washed with PBS buffer and $3 \mathrm{ml}$ fresh YNBNP plus supernatant was added. Plates were then incubated with gentle agitation on a rotary shaker at $37{ }^{\circ} \mathrm{C}$ for $36 \mathrm{~h}$. Biofilms were washed and stained with $25 \mu \mathrm{g}$ $\mathrm{ml}^{-1}$ concanavalin A-Alexa Fluor 594 conjugate (C-11253; Bioscience) for $45 \mathrm{~min}$ at $37^{\circ} \mathrm{C}$ and observed with a Zeiss LSM510 confocal scanning microscope equipped with Plan-Neofluar $\times 20$ magnification/0.5-numerical aperture (for measuring depth) and $\times 40$-magnification/1.3-numerical aperture (for image acquisition) oil objectives. A HeNe1 laser was used to excite at a $543 \mathrm{~nm}$ wavelength. All images were captured using a Zeiss LSM Image Browser.

Microtitre plate biofilm assay. Biofilm formation in C. albicans was measured in 96-well polystyrene plates (Sarstedt) essentially as described previously (Ramage et al., 2001). Cells were treated with supernatant as described above and $100 \mu \mathrm{l}$ cultures were placed in the wells of the plate for an initial incubation period of $1 \mathrm{~h}$ at $37^{\circ} \mathrm{C}$. The media and non-adherent cells were removed and the wells were washed twice with $100 \mu \mathrm{l}$ fresh growth medium to remove nonadhered cells. Fresh growth medium $(100 \mu \mathrm{l})$ plus supernatant was added to the wells, and the plates were reincubated statically for $24 \mathrm{~h}$. Biofilm formation was measured using a semiquantitative XTT (Sigma) reduction assay. Cultures with no added supernatant were used as controls. To investigate the effects of metal availability, $\mathrm{FeCl}_{3}$ was added to wells at a final concentration of $0.1 \mathrm{mM}$ and EDTA was added at a final concentration of $0.2 \mathrm{mM}$. Each experiment was repeated in triplicate with six technical replicates for each condition and control. Changes in $\mathrm{pH}$ during the assay were monitored using Panpeha $\mathrm{pH}$-indicator strips (Sigma). Means were compared using pairwise $t$-tests or, in the case of multiple treatments, by one-way ANOVA followed by least significant difference (LSD) analysis $(P=0.05)$.

RNA isolation and transcript profiling. Cultures from which RNA was extracted were grown in the presence of supernatant for $6 \mathrm{~h}$ in YNBNP, after which time, cells were harvested by centrifugation (4000 $g$ for $5 \mathrm{~min}$ ) at room temperature, washed in sterile water and immediately frozen in liquid nitrogen and stored at $-80{ }^{\circ} \mathrm{C}$. Cells were sheared mechanically with a Mikro-Dismembrator (Braun) and RNA was extracted using Trizol Reagent (GibcoBRL), as described previously (Hawser et al., 1998). Cy3- and Cy5-labelled cDNA were prepared from total RNA, and the probes were hybridized with almost whole genome microarrays containing approximately $6000 \mathrm{C}$. albicans genes (Eurogentec). Slides were scanned using a ScanArray Lite scanner (PerkinElmer Life Sciences) and quantified using QuantArray software. Data normalization and analysis were per- 
formed using GeneSpring software (Silicon Genetics) and statistical analysis was performed using significance analysis of microarrays (SAM) (Tusher et al., 2001). Expression ratios were calculated by comparing cells treated with supernatants with their untreated hyphal control. For the analysis of changes in gene expression, the transcription profiles of each test condition were compared against a reference profile generated by comparing expression in untreated yeast $\left(\right.$ YNB $\left.30{ }^{\circ} \mathrm{C}\right)$ and hyphal $\left(\right.$ YNBNP $\left.37^{\circ} \mathrm{C}\right)$ cells. Data from three independent biological replicates were used for each analysis and genes which showed a differential expression of twofold or greater at an SAM false discovery rate of $\leqslant 5 \%$ were considered significant. Complete expression data are lodged in the EBI database under accession number E-MEXP-1614.

RT-PCR amplification. For RT-PCR, treated RNA (10-15 $\mu \mathrm{g})$ was used to create cDNA using random primers (Promega) and Superscript III Reverse Transcriptase (Invitrogen) according to the manufacturer's instructions. PCRs were carried out using primer pairs designed to have the same melting temperature (Supplementary Table S2). The following conditions were used for PCR amplification: $94{ }^{\circ} \mathrm{C}$ for $4 \mathrm{~min}, 94{ }^{\circ} \mathrm{C}$ for $1 \mathrm{~min}, 5{ }^{\circ} \mathrm{C}$ for $1 \mathrm{~min}, 72{ }^{\circ} \mathrm{C}$ for $2 \mathrm{~min}$ and a final elongation step of $72{ }^{\circ} \mathrm{C}$ for $10 \mathrm{~min}$ for $26-30$ cycles depending on the primer set.

\section{RESULTS}

\section{$\boldsymbol{P}$. aeruginosa supernatants impair C. albicans biofilm development}

Microbial biofilm formation follows conserved patterns, initiating with growth and attachment of a basal layer of cells to a surface followed by development of the biofilm ultrastructure (Yeater et al., 2007). In C. albicans, this initial basal layer consists predominantly of yeast cells, with upper layers formed chiefly of hyphal cells, with some pseudohyphal and yeast cells also visible in much lesser numbers (Blankenship \& Mitchell, 2006). We tested the ability of $C$. albicans to adhere to and form biofilms on solid surfaces following treatment with $P$. aeruginosa supernatants. $C$. albicans was incubated with pieces of silicone under biofilm-permissive conditions in the presence and absence of supernatants from two homoserine lactone (HSL)producing (CF144 and PAO1) and two HSL-non-producing (CF177 and PAO1 $\Delta$ QS) isolates (Finnan et al., 2004; McAlester et al., 2008). Biofilms were examined by confocal laser scanning microscopy (CLSM), allowing both visualization of biofilms and measurement of the biofilm depth (Fig. 1). This revealed that all supernatants dramatically reduced $C$. albicans biofilm formation on silicone, clearly highlighted by the biofilm depth measurements (Fig. 1). The effect was most severe with supernatants that prevent the switch to hyphal growth (CF144 and PAO1) but was also observed with CF177 and PAO1 $\Delta$ QS supernatants, where both yeast and hyphal cells were visible. To investigate this phenomenon further, we examined $C$. albicans biofilm formation on polystyrene in the presence or absence of the supernatants using a semiquantitative assay (Ramage et al., 2001). Cells were grown in microtitre wells and, as previously reported, supernatants did not cause any reduction in total cellular growth. There was,

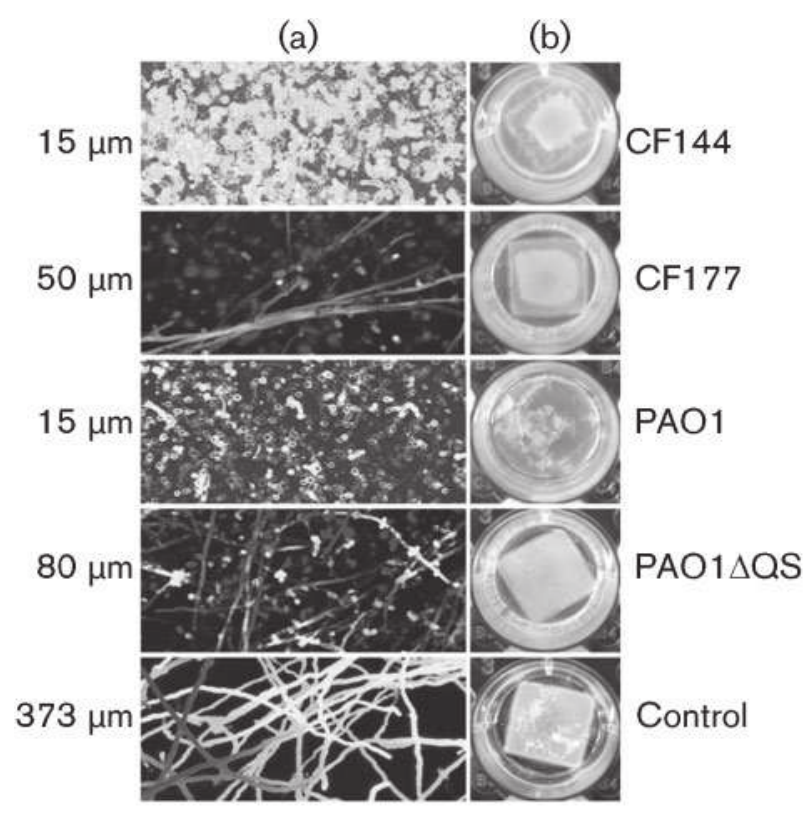

Fig. 1. CLSM images of C. albicans biofilms formed in the presence or absence of supernatant from $P$. aeruginosa strains. $C$. albicans biofilms were allowed to form on silicone squares in YNBNP $\left(37^{\circ} \mathrm{C}\right)$ for $36 \mathrm{~h}$ with/without supernatant from the indicated strain of $P$. aeruginosa and were examined by CLSM. Control refers to untreated, wild-type C. albicans (SC5314). (a) CLSM image of biofilms. (b) Silicone squares in wells prior to imaging. Biofilm depths on the surface of the silicone are shown to the left.

however, a dramatic reduction in the ability of C. albicans to form biofilms in the presence of each of the four supernatants when used at a final concentration of either $2 \times$ (Fig. $2 \mathrm{a})$ or $1 \times$ (data not shown). This phenomenon was observed using YNBNP (Fig. 2) or RPMI media (data not shown), both by quantifying cells (Fig. 2a) and by microscopic examination of the polystyrene surface, where there was a lower density of adhered cells compared with the control treatment (data not shown). There was no significant difference between the effects of supernatants from high HSL-producing strains and those from low HSLproducing strains. To determine whether this effect was specific to $P$. aeruginosa, we prepared supernatants from Escherichia coli and the environmental Pseudomonas species $P$. fluorescens. Supernatant from both of these non-clinical bacterial species reduced $C$. albicans biofilms to a similar extent as the $P$. aeruginosa supernatants (Fig. 2b). In addition, the $C$. albicans response is not strain specific, as two further wild-type strains BWP17 and DAY185 also showed the same response as SC5314 with reduced biofilm formation in the presence of $P$. aeruginosa CF177 supernatant (Fig. 2c). Interestingly, the BWP17 strain appears to have considerably reduced biofilm formation ability compared with the other wild-type strains, even in the absence of supernatant. 

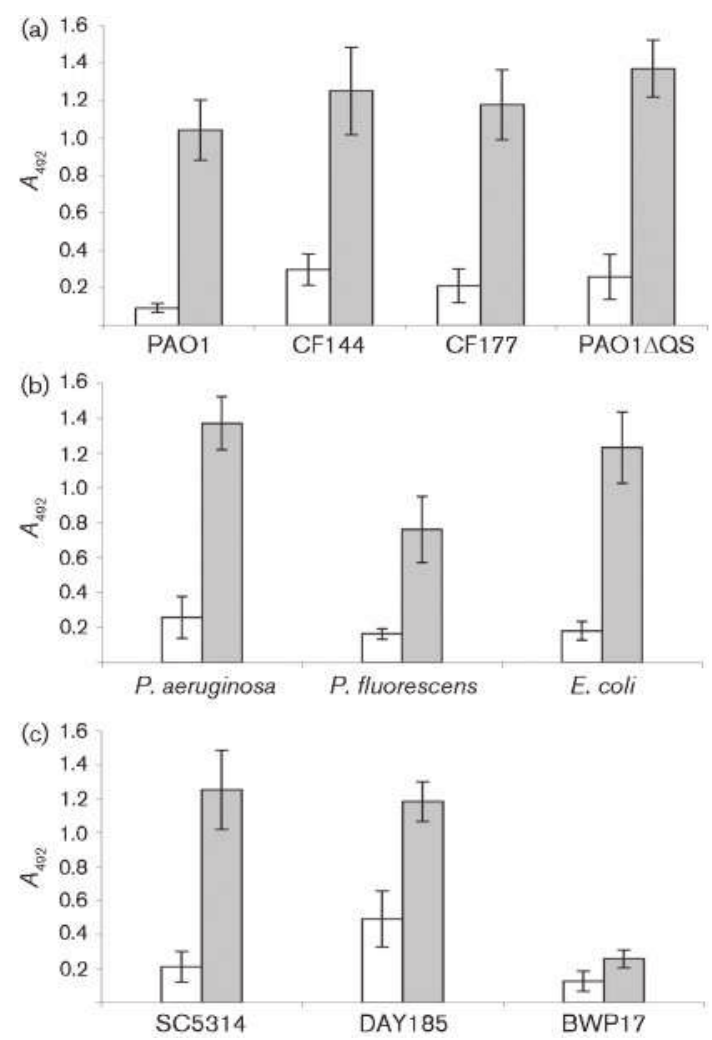

Fig. 2. Biofilm formation is reduced by the addition of bacterial supernatant. C. albicans was incubated in YNBNP medium with/ without (open/filled bars) bacterial supernatant at $37{ }^{\circ} \mathrm{C}$ in polystyrene microtitre wells. Biofilm formation was calculated after $24 \mathrm{~h}$ by using the XTT reductase assay. (a) Supernatants from both HSL-producing (CF144 and PAO1) and HSL-non-producing (CF177 and PAO1 $\triangle \mathrm{OS}$ ) $P$. aeruginosa strains reduce the ability of C. albicans SC5314 to form biofilms in the wells. (b) Supernatants from $P$. aeruginosa strain PAO1, P. fluorescens PfO-1 and E. coli $\mathrm{DH} 5 \alpha$ are also able to inhibit biofilm formation. (c) Different $C$. albicans wild-type strains exhibit reduced biofilm formation in response to supernatant from $P$. aeruginosa PAO1. Error bars show SD.

One possible reason for the observed reduction in $C$. albicans adhesion may be that addition of bacterial supernatants perturbs the fungal environment making it less optimal for biofilm formation. Initially, we investigated whether supernatants were in any way modulating $\mathrm{pH}$, as acidification of the culture media is known to negatively affect biofilm formation. Both the hyphal-inducing media YNBNP and the LB in which supernatants are prepared have a $\mathrm{pH}$ of 6.5. Even after incubation of the supernatant with cells in the polystyrene wells for $1 \mathrm{~h}$, there was no alteration in $\mathrm{pH}$, establishing that $\mathrm{pH}$ changes do not play a role here (data not shown). Another factor that is believed to play a role in hyphal development and effective biofilm formation in $C$. albicans is nutrient availability, in particular the availability of cationic compounds such as iron (Almeida et al., 2009; Baillie \& Douglas, 1998;
Hameed et al., 2008; Ramage et al., 2007). To determine if iron deficiency was involved in limiting biofilm formation we repeated the assay with an excess of iron in the media (Fig. 3). We also added the cation chelator EDTA into supernatant to give an indication of whether excess metal availability was inhibiting the development of healthy biofilms (Fig. 3). Under both conditions, C. albicans biofilm formation remained inhibited in the presence of bacterial supernatants, indicating that neither iron limitation nor excess of cationic metal compounds in the media is responsible for the effect of bacterial supernatant on biofilms.

The observed effects of supernatant could be a consequence of impaired C. albicans adhesion to solid surfaces or of impaired biofilm progression and development. To dissect the stage of adhesion/biofilm development at which supernatants have their primary effect, we modified the microtitre assay so that supernatant was only present either during the initial adhesion phase (0-1 h; condition 2) or during the biofilm development phase (1-24 h; condition 3) (Fig. 4). After $24 \mathrm{~h}$ of incubation, cells were washed and the XTT reduction assay was performed as described above. We compared these treatments to the conditions tested previously: supernatant present during the entire process (condition 1) or not present at all (condition 4). When PAO1 supernatant was only present during initial adhesion (condition 2) the biofilms formed were comparable to those in the no supernatant control (condition 4) (Fig. 4). In contrast, when supernatant was only present in the later developmental stage (condition 3) we observed a reduction in biofilm formation similar to that seen when supernatant was present throughout the

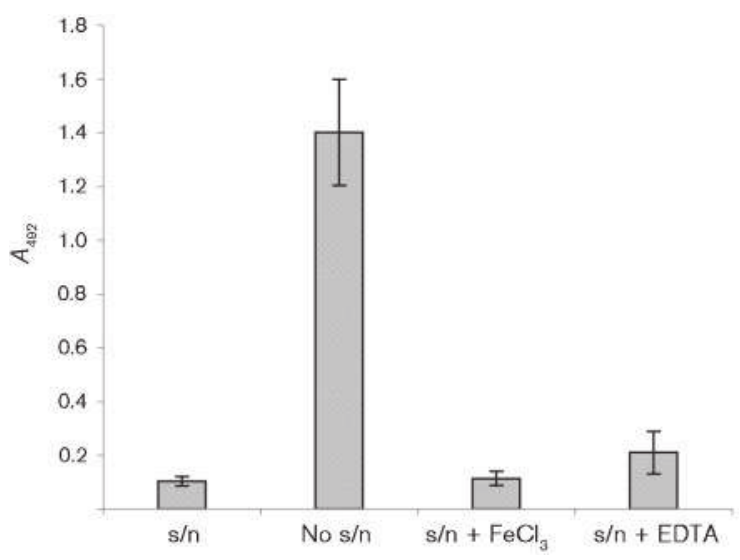

Fig. 3. $P$. aeruginosa supernatants do not influence biofilm formation by altering ion availability. C. albicans biofilm formation in polystyrene microtitre plate wells in the presence or absence of $P$. aeruginosa PAO1 supernatant was measured as described in the legend to Fig. 2. To test if an iron deficiency was limiting biofilm formation, $\mathrm{FeCl}_{3}$ was added to a final concentration of $0.1 \mathrm{mM}$. To chelate any excess ions present in the supernatant, EDTA was added to a final concentration of $0.2 \mathrm{mM}$. Error bars show SD. 


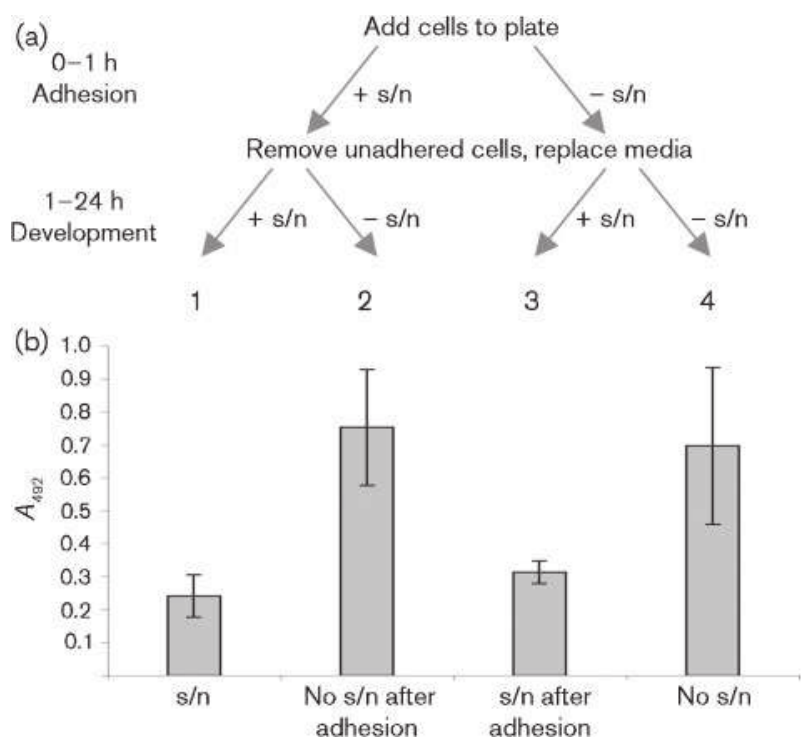

Fig. 4. $P$. aeruginosa supernatants specifically inhibit biofilm development and not initial adhesion. (a) Flow diagram indicating the experimental stages at which PAO1 supernatant was added. Condition 1 represents the typical plus supernatant incubation, with supernatant present during both adhesion $(0-1 \mathrm{~h})$ and biofilm formation (1-24 h) stages. In condition 2, C. albicans cells were only exposed to supernatant during the initial adhesion step (0$1 \mathrm{~h}$ ). For condition 3, cells were only exposed to supernatant during biofilm development (1-24 h). Condition 4 is the standard control with no supernatant. (b) C. albicans SC5314 biofilm formation in polystyrene microtitre plate wells was measured as described in the legend to Fig. 2 under the four conditions described above. Statistical analysis inidicated no significant difference between conditions 1 and 3 or between condtions 2 and 4 (one-way ANOVA followed by LSD test, $P=0.05$ ). Error bars show SD. s/n, Supernatant.

experiment (condition 1). Similar results were obtained with PAO1 $\triangle \mathrm{QS}$ supernatant (data not shown). One-way ANOVA and LSD post-hoc analyses indicate that there is no significant difference between mean levels of absorbance in conditions 1 and $3(P=0.171)$ or conditions 2 and 4 $(P=0.272)$. Therefore, controlling the stage of supernatant addition reveals that inhibition by secreted factors occurs primarily during the process of biofilm maturation.

\section{Supernatants do not impair biofilm development via interference with the yeast-hyphal morphological switch}

While the data in Fig. 4 indicate that supernatants do not mediate their effect by targeting yeast adherence, it is not clear whether they impair biofilm formation by interfering with the yeast-hyphal transition. Because $P$. aeruginosa supernatants are already known to influence the $C$. albicans morphological switch (McAlester et al., 2008), we investigated the possibility that this critical developmental stage is the target during inhibition of biofilm formation. We initially looked at supernatant effects in non-inducing YNB media. Under these conditions, cells will remain predominantly in the yeast form and have reduced capacity to form biofilms. When supernatant was added, we observed no effect on biofilm formation (Fig. 5a), consistent with our findings that yeast adherence is not being targeted (Fig. 4). In contrast, when a constitutively filamenting tup1/tup1 strain was used, supernatant addition resulted in severe inhibition of biofilm formation (Fig. 5b). Thus, even in the absence of a yeast-hyphal transition, it appears that supernatant can still impair biofilm formation in a filamenting strain. This was evident when using supernatant from either PAO1 (Fig. 5b) or the HSL-non-producing strain $\mathrm{PAO} 1 \triangle \mathrm{QS}$ (data not shown), indicating that the effect is HSL-independent. Further analysis confirmed that addition of supernatant without HSL did not have any inhibitory effect on hyphal growth in either the wild-type SC5314 or tup1/tup1 strain (data not shown).

\section{Supernatants affect $C$. albicans gene expression}

To investigate whether the phenotypic effects of supernatants on $C$. albicans were reflected in changes in gene expression, we employed a transcriptome profiling approach. This may provide insights into the mechanism(s) by which bacteria are able to modulate fungal physiology and function. As supernatants impair biofilm formation but do not appear to do this via specific targeting of yeast adhesion or the morphological switch, we chose an early time point in biofilm maturation $(6 \mathrm{~h})$ at which to study gene expression. Using the microtitre assay, we confirmed that supernatants have already begun to mediate their effect on the nascent biofilm at this time. To do this, we again used the biofilm assay but shortened the second phase of supernatant incubation from 24 to $6 \mathrm{~h}$. Although biofilm development is still at an early stage after $6 \mathrm{~h}$, PAO1 supernatant had a clear effect (Fig. 6). Consequently, a $6 \mathrm{~h}$ time point was used for all transcript profiling experiments. Supernatants from all four $P$. aeruginosa strains were analysed to investigate their effects on global gene expression in $C$. albicans under hyphalinducing conditions (YNBNP at $37^{\circ} \mathrm{C}$ ) compared with an untreated hyphal control. In these experiments, the morphology of $C$. albicans treated with supernatant from low HSL-producing strains, CF177 and PAO1 $\Delta \mathrm{QS}$, was largely hyphal $(\mathrm{H})$, and those treated with supernatant from strains CF144 and PAO1 was almost $100 \%$ yeast (Y). Hierarchical cluster analysis revealed many changes in gene expression but two clear patterns were apparent (Fig. 7). First, when compared with the reference (expression in yeast versus hyphae), the expression profiles following treatment with the four supernatants clustered together, indicating that there were many common changes as a result of addition of the bacterial supernatants. Second, within the four profiles obtained following supernatant treatment, those from the high HSL producers (CF144 and PAO1) paired together, as did those from the low producers (CF177 and PAO1 $\triangle Q S)$. This is consistent with 

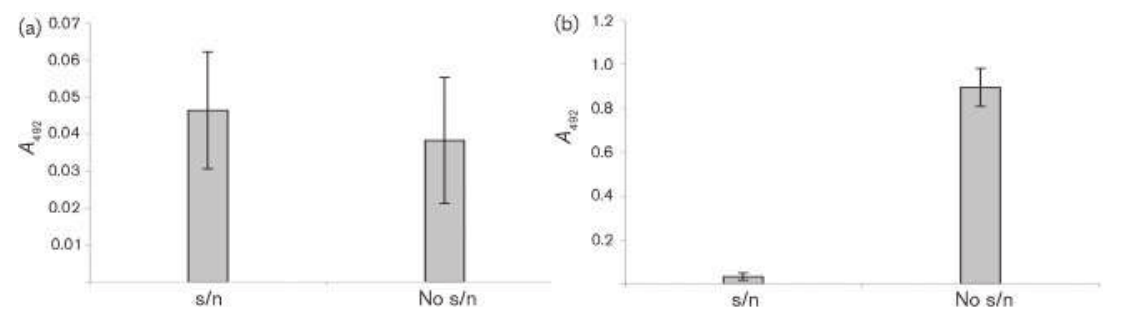

Fig. 5. Effect of $P$. aeruginosa supernatant on biofilm development is not linked to the yeasthyphal transition. C. albicans biofilm formation in polystyrene microtitre plate wells after treatment with $P$. aeruginosa $\mathrm{PAO} 1$ supernatant was measured as described in the legend to Fig. 2. (a) C. albicans SC5314 was incubated at $37{ }^{\circ} \mathrm{C}$ in non-inducing YNB media with/without supernatant. (b) The constitutively filamenting C. albicans tup1/tup1 strain BCa2-10 was incubated with or without addition of supernatant in YNBNP media at $37^{\circ} \mathrm{C}$. Error bars show SD.

the idea of specific changes in gene expression being caused by the presence of HSLs in CF144/PAO1 supernatants but also with an HSL-independent effect of all supernatants.

\section{Alterations in biofilm-linked genes}

We previously showed that supernatants containing HSLs affected the C. albicans morphological transition (McAlester et al., 2008), and these data were supported by a similar pattern of expression induced by supernatants from strains CF144 and PAO1. Furthermore, our observation of a general phenotypic effect was reflected by the large number of genes which exhibit altered expression in response to all $P$. aeruginosa supernatants. Looking at this response to supernatants from all four strains, and applying a twofold cut-off, 109 genes showed increased expression and 129 showed decreased expression relative to the reference (Supplementary Tables S3 and S4). These genes were classified using gene ontology resources on the Candida Genome Database (CGD) website and additional information where available. Many of the differentially regulated genes were involved in nucleic acid binding,

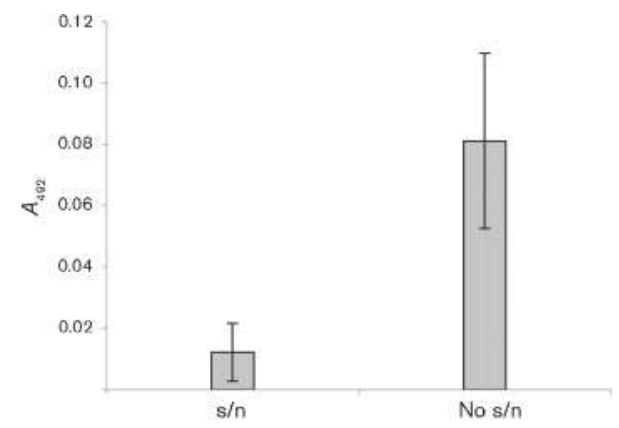

Fig. 6. Inhibition by supernatant can be observed at early stages of C. albicans biofilm development. C. albicans SC5314 biofilm formation with or without addition of $P$. aeruginosa PAO1 supernatant was measured as described in the legend for Fig. 2, except the XTT reductase assay was performed after only $6 \mathrm{~h}$ incubation. Error bars show SD. ribosome activity and transport, suggesting a cellular response to changing environmental conditions but there was no evidence of global stress-related signal transduction pathways being activated. This is consistent with our previous report that these $P$. aeruginosa supernatants do not have any negative impact on growth rates (McAlester et al., 2008). The most striking class of upregulated genes encode proteins that are believed to be involved in drug export, for example $S N Q 2$ and $C D R 11$, which suggests that the supernatants contain metabolites perceived as detrimental by Candida (Supplementary Table S3). With respect to downregulated genes, the expression of $C$. albicans genes associated with adhesion or biofilm properties was reduced (Supplementary Table S4). To explore this in more detail, we compiled expression data for three classes of genes: those reported in various studies to regulate or be involved in biofilm formation (Nobile \& Mitchell, 2006), the ALS gene family that encodes surface proteins involved in adhesion (Hoyer et al., 2008) and the RBT genes, which have been defined as 'repressed by TUP1' (Braun et al., 2000) (Table 1). Important regulators such as CHK1 and TEC1, which have also been shown to affect biofilm formation, showed decreased expression across all treatments. In contrast with the downregulated expression of these biofilm formation genes, there was a strong increase in expression of $Y W P 1$, which encodes a protein that is associated with biofilm dispersal (Granger et al., 2005). The pattern of expression of the ALS genes, in particular the reduced expression of ALS1, ALS2 and ALS3, suggests that Pseudomonas supernatants may in fact be reducing adherence in $C$. albicans. Note that the revised annotation of the C. albicans genome sequence means that both CA0448 and CA0519 on the microarray represent ALS3 (Hoyer et al., 2008). Furthermore, the consistent downregulation of $R B T$ genes is also notable, especially knowing that RBT1, RBT5 and RBT8 encode cell wall/ surface proteins that are typically induced during the switch from yeast to hyphal growth. To verify array data, semiquantitative RT-PCR was performed on genes with the highest differential expression or with functions related to biofilm formation, adhesion and morphological state (Fig. 8). This confirmed the increase in expression in drug 


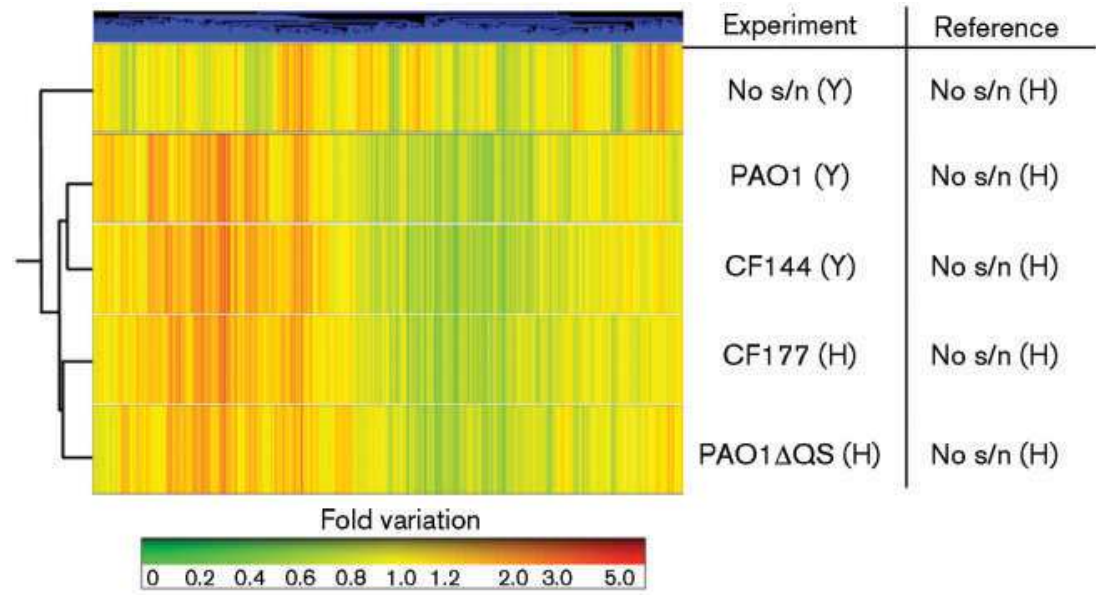

Fig. 7. Hierarchical cluster analysis of the global transcriptional response of C. albicans to supernatants from $P$. aeruginosa. C. albicans genes were clustered based on their expression profiles. Ratios between the experimental and reference samples are represented by a green to red colour scale. The yeast $(\mathrm{Y})$ or hyphal $(\mathrm{H})$ morphology of the Candida cells following the addition of supernatant is indicated. No $\mathrm{s} / \mathrm{n}$ refers to untreated C. albicans SC5314. Other than the yeast control condition [no s/n (Y)] YNB $\left(30{ }^{\circ} \mathrm{C}\right)$, all experiments were carried out in YNBNP medium at $37^{\circ} \mathrm{C}$. efflux genes such as SNQ2 and CDR2 and the decrease in expression of the key genes RBT1, RBT4 and ALS3. As expected, $H W P 1$ is only expressed in cells growing as hyphae in both the control experiment and after supernatant treatment. However, the 'yeast-specific' gene YWP1

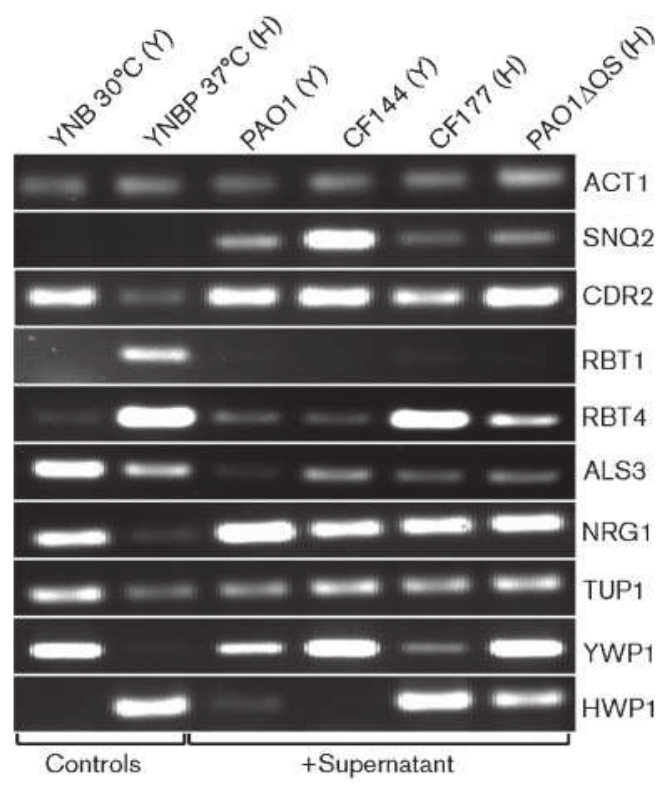

Fig. 8. Confirmatory RT-PCR analysis. Changes in the levels of expression of selected $C$. albicans genes following treatment with $P$. aeruginosa supernatants were determined by semiquantitative RT-PCR. The genes tested included those proposed to be involved in drug export (SNQ2, CDR2), surface properties (RBT1, HWP1, RBT4, ALS3), morphology (NRG1, TUP1) and biofilm inhibition (YWP1). ACT1 was used as a control. The yeast $(\mathrm{Y})$ or hyphal $(\mathrm{H})$ morphology of the Candida cells following the addition of supernatant is indicated. Other than the control condition YNB $30^{\circ} \mathrm{C}$, all experiments were carried out $6 \mathrm{~h}$ after transfer of the cultures from YNB $\left(30{ }^{\circ} \mathrm{C}\right)$ to YNBNP $\left(37^{\circ} \mathrm{C}\right)$ medium containing the bacterial supernatant indicated. shows an interesting, morphology-independent increase in expression in response to bacterial supernatants. RBT1 and RBT4 fail to show the expected degree of increase in expression in hyphae. Interestingly, the modest increase in expression of the hyphal repressors TUP1 and NRG1 that was seen in the array data for some treatments was confirmed. Increased expression of these morphogenetic repressors, and of the biofilm repressor, YWP1, is predicted to have a significant negative impact on the capacity of $C$. albicans to form mature biofilms.

\section{DISCUSSION}

Pseudomonas-Candida interactions are emerging as a complex theme with possible implications for human health. Based on previous work, it is clear that some of these interactions are mediated by secreted signal molecules, namely bacterial HSLs and the Candida molecule farnesol. Previously, we showed that addition of supernatants from HSL-producing P. aeruginosa strains (CF144, $\mathrm{PAO} 1)$ to $C$. albicans inhibited hyphal development, whereas addition of supernatant from non-HSL-producing strains (CF177, PAO1 $\Delta$ QS) had no morphological effect (McAlester et al., 2008). These effects were not accompanied by an impact on growth. Although the mechanism remains unknown, recent data suggest that this effect could operate via the RAS-cAMP protein kinase A signal transduction pathway (Davis-Hanna et al., 2008). Our focus in this study, however, was on other virulence-related effects of $P$. aeruginosa supernatants on $C$. albicans. Because we analysed supernatants from three independent $P$. aeruginosa strains - two clinical isolates (CF144 and CF177) and the laboratory strain PAO1 - and included two HSL-producing (PAO1 and CF144) and two nonHSL-producing (PAO1 $\triangle \mathrm{QS}$ and CF177) strains, we are confident that the observed effects are HSL-independent and of general significance.

It was striking from our phenotypic studies that supernatants from all four $P$. aeruginosa strains negatively 
Table 1. Fold changes in expression of genes implicated in biofilm formation and/or adhesion after treatment with supernatant from four $P$. aeruginosa strains

\begin{tabular}{|c|c|c|c|c|c|}
\hline \multirow[t]{2}{*}{ Gene name $(\mathrm{CGD})^{\star}$} & \multirow[t]{2}{*}{ Accession no. } & \multicolumn{4}{|c|}{ Average ratios $\dagger$} \\
\hline & & PAO1 (Y) & CF144 (Y) & CF177 (H) & PAO1 $\triangle Q S(H)$ \\
\hline \multicolumn{6}{|c|}{ Genes relevant to biofilm formation: } \\
\hline EFG1 & CA2787 & 0.77 & 0.88 & 0.85 & 1.33 \\
\hline KEM1 & CA0767 & 0.9 & 1.1 & 1.0 & 1.1 \\
\hline MDS3 & CA5937 & 1.0 & 1.1 & 1.12 & 1.16 \\
\hline SUV3 & CA2908 & 1.8 & 2.14 & 2.1 & 1.5 \\
\hline$B C R 1$ & CA3685 & 0.7 & 0.9 & 0.9 & 1.1 \\
\hline TEC1 & CA6138 & 0.32 & 0.38 & 0.58 & 0.43 \\
\hline YWP1 & CA1678 & 6.7 & 6.0 & 3.6 & 8.7 \\
\hline MDR1 & CA2968 & 0.8 & 0.88 & 1.0 & 0.97 \\
\hline$C D R 1$ & CA6066 & 2.1 & 1.7 & 1.85 & 1.5 \\
\hline$C D R 2$ & CA6099 & 2.5 & 2.5 & 1.7 & 1.1 \\
\hline NUP85 & CA6152 & 1.1 & 1.0 & 1.3 & 1.1 \\
\hline CHK1 & CA4676 & 0.2 & 0.18 & 0.2 & 0.2 \\
\hline $\mathrm{CPH} 1$ & CA0154 & 0.73 & 0.85 & 0.87 & 0.95 \\
\hline EAP1 & CA3173 & 1.1 & 0.9 & 0.7 & 0.8 \\
\hline MKC1 & CA5865 & 0.68 & 0.65 & 1.1 & 0.93 \\
\hline GCN4 & CA3126 & 0.33 & 0.3 & 0.31 & 0.36 \\
\hline \multicolumn{6}{|l|}{ RBT genes: } \\
\hline$R B T 1$ & CA2830 & 0.14 & 0.05 & 0.15 & 0.1 \\
\hline$R B T 2$ & CA3957 & 0.28 & 0.27 & 0.27 & 0.57 \\
\hline RBT4 & CA0104 & 0.23 & 0.17 & 0.31 & 0.25 \\
\hline RBT5 & CA2558 & 0.38 & 0.81 & 0.4 & 0.92 \\
\hline RBT7 & CA0169 & 0.99 & 0.97 & 0.97 & 0.88 \\
\hline$R B T 8$ & CA4381 & 0.71 & 0.11 & 0.57 & 1.18 \\
\hline \multicolumn{6}{|l|}{ ALS gene family: } \\
\hline ALS1 & CA0316 & 0.87 & 1.05 & 0.43 & 0.38 \\
\hline ALS2 & CA0413 & 1.02 & 0.94 & 1.0 & 0.99 \\
\hline$A L S 3$ & CA0509 & 0.48 & 0.75 & 0.69 & 0.94 \\
\hline ALS3 & CA0448 & 0.16 & 0.15 & 0.30 & 0.18 \\
\hline ALS4 & CA5173 & 0.64 & 0.66 & 0.50 & 0.67 \\
\hline ALS5 & CA2852 & 1.27 & 1.59 & 1.12 & 1.15 \\
\hline ALS6 & CA5713 & 1.01 & 1.14 & 1.11 & 1.14 \\
\hline ALS7 & CA5699 & 1.22 & 1.19 & 1.15 & 1.05 \\
\hline ALS9 & CA1426 & 1.14 & 1.94 & 0.90 & 0.92 \\
\hline
\end{tabular}

${ }^{*}$ Genes exhibiting a significant change in expression in response to all supernatants are given in bold type.

$†$ Significant changes in gene expression given in bold type (twofold cut off; false discovery rate $\leqslant 5 \%$ ).

affected biofilm formation. Further dissection revealed that it is biofilm maturation which is targeted by supernatants and not adhesion or the yeast-hyphal morphological transition. Although the role of Tup1 in morphogenesis has not yet been elucidated fully, the discovery that supernatant from the non-HSL-producing PAO1 $1 \mathrm{QS}$ strain could severely inhibit biofilm development in the constitutively filamenting tup1/tupl strain supports the idea that we are seeing an additional effect on C. albicans morphology which is unrelated to that of HSLs.

Our microarray analysis, which was performed in the early stages of the interaction, provides a broad view of how supernatants influence fungal physiology and may also give indirect insights into mechanisms involved in biofilm formation. Concurrent with our phenotypic study, the microarray revealed two distinct patterns. Firstly, the profiles of the HSL-producing strains clustered together, as did those from the non-HSL-producing strains, revealing a clear HSL-related effect on gene expression. More importantly, we saw a dramatic global effect, with all supernatants affecting expression of a common set of 238 genes, irrespective of whether the Candida was growing as yeast $(\mathrm{CF} 144 / \mathrm{PAO} 1)$ or hyphae $(\mathrm{CF} 177 / \mathrm{PAO} 1 \Delta \mathrm{QS})$. Most of these affected genes were related to growth or metabolism but the two key observations were the increase in expression of drug export-type genes and the alterations predicted to negatively affect adhesion and biofilm 
formation. This latter group included genes that are required for biofilm formation, adhesins and negative regulators of hyphal development, including NRG1 and the YWP1 gene.

Although it is not possible at this stage to establish the relative importance of each individual change in gene expression, genetic studies with YWP1 suggest that the morphology-independent increase in expression of this gene may be significant. C. albicans strains lacking YWP1 have been shown to display greater adhesion and biofilm formation, implying a role for Ywplp in biofilm dispersal. However, analysis of adhesion/biofilm formation in a $C$. albicans ywp1 null strain revealed that deletion of this gene alone was not sufficient to ameliorate the responses to $P$. aeruginosa supernatants (data not shown). The upregulation of the hyphal repressors NRG1 and TUP1 may also be of significance as these genes are both components of a pathway controlling expression of downstream targets involved in hyphal formation (Braun et al., 2001). Interestingly, supernatants were still able to severely impair biofilm development in a tup 1/tup 1 mutant, indicating that this gene is not the key target. An alternative explanation is that reduced expression of other key genes in biofilm formation, namely the ALS adhesins (Hoyer et al., 2008) or the regulators of biofilm formation CHK1 and TEC1, is responsible for the phenomenon. It is most likely that changes in C. albicans adhesion and biofilm formation are, not unsurprisingly, the result of a co-ordinated alteration in the expression of a cohort of genes. Although the effects of $P$. aeruginosa supernatants on Candida biofilm formation are independent of HSLs, it remains to be determined whether induced alterations in Candida farnesol levels play some role in the adherence and biofilm defect. This is an attractive hypothesis as farnesol is known to inhibit biofilm formation. However, as a counter-argument, farnesol is believed to operate via Chk1p (Kruppa et al., 2004) and CHK1 expression is strongly reduced by supernatants, suggesting that a role for this pathway is less likely.

Several plausible explanations can be put forward for the observed phenomena and identification of the component(s) of the $P$. aeruginosa supernatant that reduces biofilm formation will be crucial in dissecting the mechanistic basis of this interaction. The main possibilities are either production of a secreted metabolite or alterations in the levels of particular nutrients or macromolecules. Either scenario is equally relevant because the external factors that reduce $C$. albicans biofilm maturation are not currently known. As a possible corollary, synergistic interactions between the bacterium Pseudomonas putida and the yeast S. cerevisiae have also been reported (Romano \& Kolter, 2005). In the case of the $P$. aeruginosa-C. albicans interaction, changes in $\mathrm{pH}$ or routine media components have been discounted. We also ruled out the possibility that the observed phenotype was due to a limitation in the availability of iron, such as that which may occur as a result of scavenging by siderophores potentially present in bacterial supernatants. Interestingly, supernatants from an E. coli and a $P$. fluorescens strain were also able to reduce adhesion to polystyrene, indicating that the component(s) responsible may be common in bacterial supernatants. A possible contender for this role could be bacterial lipo/ exopolysaccharide or other lipid molecules. A recent study in $P$. aeruginosa has identified an unsaturated fatty acid (cis2-decenoic acid) that has the capacity to disperse the biofilms formed by a range of microbes (Davies \& Marques, 2009). It will be interesting to identify whether this small lipid messenger is also present in supernatants from other bacterial species and if it has a role in the cross-species communications observed here.

The importance of bacteria-Candida interactions in medical contexts has been recognized previously (El-Azizi et al., 2004; Nseir et al., 2007; Wargo \& Hogan, 2006). Candida can colonize epithelia causing problems such as thrush and several studies have examined effects of lactobacilli on C. albicans. Results are conflicting, with some studies reporting general antifungal activity of lactobacilli and others finding that antifungal activity is uncommon (Falagas et al., 2006; Strus et al., 2005a, b). These studies generally focused on growth inhibition, which is distinct from the phenomenon reported here that occurs without any negative impact on growth. In addition, however, it was found that some lactobacilli (Osset et al., 2001), and a single probiotic strain of E. coli (Trautner et al., 2003), could reduce Candida adherence to epithelial cells and the surface of a catheter, respectively. Recently, secreted molecules from Salmonella enterica serovar Typhimurium have been shown to reduce $C$. albicans viability, filamentation and biofilm formation in vitro and to inhibit filamentation in a Caenorhabditis elegans co-infection model (Tampakakis et al., 2009). Inhibition of C. albicans growth by $P$. aeruginosa secondary metabolites such as pyrrolnitrin or phenazines has also been reported previously. When the direct effects of $P$. aeruginosa on $C$. albicans were assessed, it was found that $P$. aeruginosa bacteria adhere to and inhibit the growth of C. albicans hyphal (but not yeast) cells (Hogan \& Kolter, 2002), and that C. albicans hyphal growth and biofilm formation is inhibited in co-culture with $P$. aeruginosa (Thein et al., 2006). The strategy in this study of using culture supernatants that lack growth inhibitory metabolites facilitated the identification of a new class of interaction, namely specific inhibition of biofilm formation.

The interaction between $C$. albicans and $P$. aeruginosa reported here may have implications for in vivo interactions of these microbes during infections of burn wounds or in the CF lung. In addition, the ability to prevent or disrupt C. albicans biofilm formation on catheters and medical implants could have useful clinical applications. The key challenges now are to identify the bacterial factor responsible for reducing biofilm formation and the fungal pathway that is targeted to mediate this effect. As this is clearly a multi-faceted interaction, it is likely that a systems approach will be required to achieve these goals. 


\section{ACKNOWLEDGEMENTS}

We thank Pat Higgins for excellent technical support, Drs Aaron Mitchell and Scott Filler for helpful discussions and Drs Mitchell, Granger and Johnson for strains. Research in J.P. M.'s and F. O.'s laboratories is supported by grants awarded by the European Union (TRAMWAYS and MICROMAIZE: FP6\#O36314), Science Foundation of Ireland (04/BR/B0597, 07/IN.1/B948, 08-RFP-GEN1319 and 08/RFP/GEN1295), the Health Research Board (RP/2006/271 and RP/ 2007/290), the Marine SSTI programme and the Department of Agriculture (FIRM grants 06RDC459 and 06RDC506, and RSF grants 06-321 and 06-377). N.A.R.G. acknowledges support from the Wellcome Trust (080088) and G. McA. thanks the SGM for travel support from the President's fund.

\section{REFERENCES}

Adams, C., Morris-Quinn, M., McConnell, F., West, J., Lucey, B., Shortt, C., Cryan, B., Watson, J. B. \& O'Gara, F. (1998). Epidemiology and clinical impact of Pseudomonas aeruginosa infection in cystic fibrosis using AP-PCR fingerprinting. J Infect 37, 151-158.

Almeida, R. S., Wilson, D. \& Hube, B. (2009). Candida albicans iron acquisition within the host. FEMS Yeast Res 9, 1000-1012.

Baillie, G. S. \& Douglas, L. J. (1998). Iron-limited biofilms of Candida albicans and their susceptibility to amphotericin B. Antimicrob Agents Chemother 42, 2146-2149.

Baillie, G. S. \& Douglas, L. J. (1999). Role of dimorphism in the development of Candida albicans biofilms. J Med Microbiol 48, 671679.

Bakare, N., Rickerts, V., Bargon, J. \& Just-Nubling, G. (2003). Prevalence of Aspergillus fumigatus and other fungal species in the sputum of adult patients with cystic fibrosis. Mycoses 46, 19-23.

Blankenship, J. R. \& Mitchell, A. P. (2006). How to build a biofilm: a fungal perspective. Curr Opin Microbiol 9, 588-594.

Braun, B. R., Head, W. S., Wang, M. X. \& Johnson, A. D. (2000). Identification and characterization of TUP1-regulated genes in Candida albicans. Genetics 156, 31-44.

Braun, B. R., Kadosh, D. \& Johnson, A. D. (2001). NRG1, a repressor of filamentous growth in C. albicans is down-regulated during filament induction. $Е M B O \mathrm{~J}$ 20, 4753-4761.

Cugini, C., Calfee, M. W., Farrow, J. M., III, Morales, D. K., Pesci, E. C. \& Hogan, D. A. (2007). Farnesol, a common sesquiterpene, inhibits PQS production in Pseudomonas aeruginosa. Mol Microbiol 65, 896906.

Davies, D. G. \& Marques, C. N. H. (2009). A fatty acid messenger is responsible for inducing dispersion in microbial biofilms. J Bacteriol 191, 1393-1403.

Davis-Hanna, A., Piispanen, A. E., Stateva, L. I. \& Hogan, D. A. (2008). Farnesol and dodecanol effects on the Candida albicans Ras1cAMP signalling pathway and the regulation of morphogenesis. Mol Microbiol 67, 47-62.

d'Enfert, C. (2006). Biofilms and their role in the resistance of pathogenic Candida to antifungal agents. Curr Drug Targets 7, 465470 .

De Sordi, L. \& Mühlschlegel, F. A. (2009). Quorum sensing and fungal-bacterial interactions in Candida albicans: a communicative network regulating microbial coexistence and virulence. FEMS Yeast Res 9, 990-999.

Ding, C. \& Butler, G. (2007). Development of a gene knockout system in Candida parapsilosis reveals a conserved role for BCR1 in biofilm formation. Eukaryot Cell 6, 1310-1319.
El-Azizi, M. A., Starks, S. E. \& Khardori, N. (2004). Interactions of Candida albicans with other Candida spp. and bacteria in the biofilms. J Appl Microbiol 96, 1067-1073.

Falagas, M. E., Betsi, G. I. \& Athanasiou, S. (2006). Probiotics for prevention of recurrent vulvovaginal candidiasis: a review. J Antimicrob Chemother 58, 266-272.

Finnan, S., Morrissey, J. P., O'Gara, F. \& Boyd, E. F. (2004). Genome diversity of Pseudomonas aeruginosa isolates from cystic fibrosis patients and the hospital environment. J Clin Microbiol 42, 57835792.

Gow, N. A., Brown, A. J. \& Odds, F. C. (2002). Fungal morphogenesis and host invasion. Curr Opin Microbiol 5, 366-371.

Gupta, N., Haque, A., Mukhopadhyay, G., Naryan, R. P. \& Prasad, R. (2005). Interactions between bacteria and Candida in the burn wound. Burns 31, 375-378.

Hameed, S., Prasad, T., Banerjee, D., Chandra, A., Mukhopadhyay, C. K., Goswami, S. K., Lattif, A. A., Chandra, J., Mukherjee, P. K. \& other authors (2008). Iron deprivation induces EFG1-mediated hyphal development in Candida albicans without affecting biofilm formation. FEMS Yeast Res 8, 744-755.

Hawser, S. P., Baillie, G. S. \& Douglas, L. J. (1998). Production of extracellular matrix by Candida albicans biofilms. J Med Microbiol 47, 253-256.

Hogan, D. A. \& Kolter, R. (2002). Pseudomonas-Candida interactions: an ecological role for virulence factors. Science 296, 2229-2232.

Hogan, D. A., Vik, A. \& Kolter, R. (2004). A Pseudomonas aeruginosa quorum-sensing molecule influences Candida albicans morphology. Mol Microbiol 54, 1212-1223.

Hornby, J. M., Jensen, E. C., Lisec, A. D., Tasto, J. J., Jahnke, B., Shoemaker, R., Dussault, P. \& Nickerson, K. W. (2001). Quorum sensing in the dimorphic fungus Candida albicans is mediated by farnesol. Appl Environ Microbiol 67, 2982-2992.

Hoyer, L. L., Green, C. B., Oh, S. H. \& Zhao, X. (2008). Discovering the secrets of the Candida albicans agglutinin-like sequence (ALS) gene family - a sticky pursuit. Med Mycol 46, 1-15.

Kerr, J. R. (1994). Suppression of fungal growth exhibited by Pseudomonas aeruginosa. J Clin Microbiol 32, 525-527.

Kojic, E. M. \& Darouiche, R. O. (2004). Candida infections of medical devices. Clin Microbiol Rev 17, 255-267.

Kruppa, M., Krom, B. P., Chauhan, N., Bambach, A. V., Cihlar, R. L. \& Calderone, R. A. (2004). The two-component signal transduction protein Chklp regulates quorum sensing in Candida albicans. Eukaryot Cell 3, 1062-1065.

McAlester, G., O'Gara, F. \& Morrissey, J. P. (2008). Signal-mediated interactions between Pseudomonas aeruginosa and Candida albicans. J Med Microbiol 57, 563-569.

Nobile, C. J. \& Mitchell, A. P. (2006). Genetics and genomics of Candida albicans biofilm formation. Cell Microbiol 8, 1382-1391.

Nobile, C. J., Andes, D. R., Nett, J. E., Smith, F. J., Yue, F., Phan, Q. T., Edwards, J. E., Filler, S. G. \& Mitchell, A. P. (2006). Critical role of Bcr1-dependent adhesins in C. albicans biofilm formation in vitro and in vivo. PLoS Pathog 2, e63.

Nseir, S., Jozefowicz, E., Cavestri, B., Sendid, B., Di Pompeo, C., Dewavrin, F., Favory, R., Roussel-Delvallez, M. \& Durocher, A. (2007). Impact of antifungal treatment on Candida-Pseudomonas interaction: a preliminary retrospective case-control study. Intensive Care Med 33, 137-141.

Osset, J., Garcia, E., Bartolome, R. M. \& Andreu, A. (2001). Role of Lactobacillus as protector against vaginal candidiasis. Med Clin (Barc) 117, 285-288. 
Ramage, G., Vande Walle, K., Wickes, B. L. \& Lopez-Ribot, J. L. (2001). Standardized method for in vitro antifungal susceptibility testing of Candida albicans biofilms. Antimicrob Agents Chemother 45, 2475-2479.

Ramage, G., Saville, S. P., Wickes, B. L. \& Lopez-Ribot, J. L. (2002a). Inhibition of Candida albicans biofilm formation by farnesol, a quorum-sensing molecule. Appl Environ Microbiol 68, 5459-5463.

Ramage, G., VandeWalle, K., Lopez-Ribot, J. L. \& Wickes, B. L. (2002b). The filamentation pathway controlled by the Efg1 regulator protein is required for normal biofilm formation and development in Candida albicans. FEMS Microbiol Lett 214, 95-100.

Ramage, G., Wickes, B. L. \& Lopez-Ribot, J. L. (2007). Inhibition on Candida albicans biofilm formation using divalent cation chelators (EDTA). Mycopathologia 164, 301-306.

Romano, J. D. \& Kolter, R. (2005). Pseudomonas-Saccharomyces interactions: influence of fungal metabolism on bacterial physiology and survival. J Bacteriol 187, 940-948.

Shiner, E. K., Rumbaugh, K. P. \& Williams, S. C. (2005). Interkingdom signaling: deciphering the language of acyl homoserine lactones. FEMS Microbiol Rev 29, 935-947.

Storey, D. G., Ujack, E. E., Rabin, H. R. \& Mitchell, I. (1998). Pseudomonas aeruginosa lasR transcription correlates with the transcription of las $A$, las $B$, and tox $A$ in chronic lung infections associated with cystic fibrosis. Infect Immun 66, 2521-2528.

Strus, M., Brzychczy-Wloch, M., Kucharska, A., Gosiewski, T. \& Heczko, P. B. (2005a). Inhibitory activity of vaginal Lactobacillus bacteria on yeasts causing vulvovaginal candidiasis. Med Dosw Mikrobiol 57, 7-17.

Strus, M., Kucharska, A., Kukla, G., Brzychczy-Wloch, M., Maresz, K. \& Heczko, P. B. (2005b). The in vitro activity of vaginal Lactobacillus with probiotic properties against Candida. Infect Dis Obstet Gynecol 13, 69-75.

Tampakakis, E., Peleg, A. Y. \& Mylonakis, E. (2009). Interaction of Candida albicans with an intestinal pathogen Salmonella enterica serovar Typhimurium. Eukaryot Cell 8, 732-737.

Thein, Z. M., Samaranayake, Y. H. \& Samaranayake, L. P. (2006). Effect of oral bacteria on growth and survival of Candida albicans biofilms. Arch Oral Biol 51, 672-680.

Trautner, B. W., Hull, R. A. \& Darouiche, R. O. (2003). Escherichia coli 83972 inhibits catheter adherence by a broad spectrum of uropathogens. Urology 61, 1059-1062.

Tusher, V. G., Tibshirani, R. \& Chu, G. (2001). Significance analysis of microarrays applied to the ionizing radiation response. Proc Natl Acad Sci U S A 98, 5116-5121.

Valenza, G., Tappe, D., Turnwald, D., Frosch, M., König, C., Hebestreit, H. \& Abele-Horn, M. (2008). Prevalence and antimicrobial susceptibility of microorganisms isolated from sputa of patients with cystic fibrosis. J Cyst Fibros 7, 123-127.

Wargo, M. J. \& Hogan, D. A. (2006). Fungal-bacterial interactions: a mixed bag of mingling microbes. Curr Opin Microbiol 9, 359364.

Whiteway, M. \& Oberholzer, U. (2004). Candida morphogenesis and host-pathogen interactions. Curr Opin Microbiol 7, 350357.

Yeater, K. M., Chandra, J., Cheng, G., Mukherjee, P. K., Zhao, X., Rodriguez-Zas, S. L., Kwast, K. E., Ghannoum, M. A. \& Hoyer, L. L. (2007). Temporal analysis of Candida albicans gene expression during biofilm development. Microbiology 153, 2373-2385. 